\title{
Going with the flow (of heat)
}

Phys. Rev. Appl. 11, 054008 (2019)
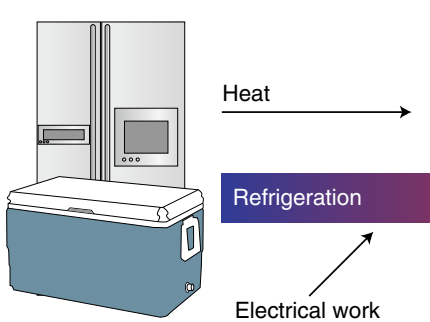

\section{Refrigeration}

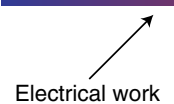

Most cooling devices operate against the natural flow of heat from hot to cold, keeping, for example, something colder than its surrounding. Yet many applications require an already favourable heat transfer from a hot device, such as a computer processor, to be accelerated. This regime, called active cooling, places very different demands on thermoelectric coolers, which use electrical work to transfer heat via the Peltier effect. Normally, Peltier coolers use materials that have a high thermoelectric power factor (the amount of heat energy than can be carried by a current) and a low thermal conductivity to reduce the reverse flow of heat from the hotter reservoir. Mona Zebarjadi, Joseph Heremans and colleagues have now developed Peltier coolers based on materials that combine a high power factor with a high thermal conductivity in order to make more effective active coolers.
Passive heat sink

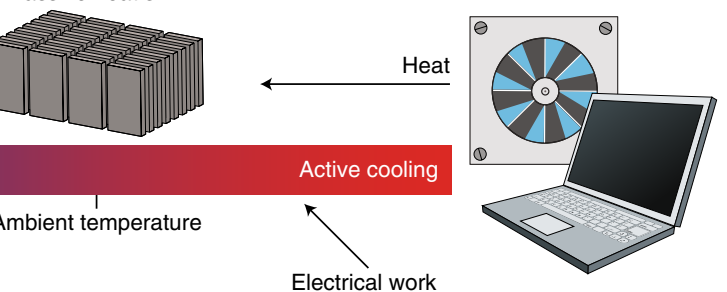

The researchers - who are based at Ohio State University and the University of Virginia - constructed their coolers using metals that, in additional to being naturally conductive, also had high power factors due to magnon drag (cobalt) or electron correlation effects (cerium-palladium). The passive thermal conductivity of their $\mathrm{Co}-\mathrm{CePd}_{3}$ device for a one kelvin heat gradient was 40 watts per metre-kelvin. When a current was applied the effective conductivity, combining both passive and thermoelectric contributions, became 1,000 watts per metre-kelvin. This device could alternate between passive and active operation to meet varying demands of heat removal in electrical devices.

Matthew Parker

Published online: 17 June 2019

https://doi.org/10.1038/s41928-019-0267-5 